\title{
FABP4-mediated homocysteine-induced cholesterol accumulation in THP-1 monocyte-derived macrophages and the potential epigenetic mechanism
}

\author{
YIDENG JIANG $^{1 *}$, SHENGCHAO MA ${ }^{2 *}$, HUIPING ZHANG ${ }^{3 *}$, XIAOLING YANG ${ }^{2}$, GUAN JUN LU ${ }^{3}$, \\ HUI ZHANG ${ }^{2}$, YANGYANG $\mathrm{HE}^{2}$, FANQI KONG ${ }^{2}$, ANNING YANG ${ }^{2}, \mathrm{HUA} \mathrm{XU}^{2}$, MINGHAO ZHANG ${ }^{2}$, \\ YUN JIAO $^{3}$, GUIZHONG LI ${ }^{2}$, JUN CAO ${ }^{2}$, YUEXIA JIA ${ }^{1}$, SHAOJU JIN $^{1}$, JUN WEI $^{3 *}$ and YINGKANG SHI ${ }^{*}$ \\ ${ }^{1}$ Department of Cardiothoracic Surgery, West China Hospital of Sichuan University, Chengdu, Sichuan 610000; \\ ${ }^{2}$ School of Basic Medical Sciences, Ningxia Medical University; ${ }^{3}$ Medical Experimental Center, \\ General Hospital of Ningxia Medical University, Yinchuan, Ningxia 750004, P.R. China
}

Received June 5, 2015; Accepted April 11, 2016

DOI: $10.3892 / \mathrm{mmr} .2016 .5315$

\begin{abstract}
Hyperhomocysteinemia (HHcy) is an independent risk factor for the development of atherosclerosis (AS), according to overwhelming number of clinical and epidemiological studies. However, the underlying pathogenic molecular mechanisms by which HHcy promotes AS remain to be fully elucidated. Fatty acid binding protein 4 (FABP4) has been shown to be important in macrophage cholesterol trafficking. The objective of the present study was to determine whether homocysteine (Hcy) accelerates AS through regulating FABP4, and then mediates cholesterol accumulation in macrophages. Hcy concentrations of $0,50,100,200$ and $500 \mu \mathrm{M}$, and $100 \mu \mathrm{M}$ Hcy $+30 \mu \mathrm{M}$ vitamin $\mathrm{B}_{12}\left(\mathrm{VB}_{12}\right)+30 \mu \mathrm{M}$ folic acid (FA) were respectively added to cultured THP-1 monocyte-derived macrophages for $24 \mathrm{~h}$. The levels of FABP4, which acts as a key factor connecting cellular lipid accumulation to inflammation, were determined using reverse transcription-quantitative polymerase chain reaction (RT-qPCR) and western blot analyses in the macrophages. The present study used a nested touchdown methylation-specific PCR assay to detect the DNA methylation status of the $F A B P 4$ promoter region. In addition, the FABP4 gene fragment was inserted into the
\end{abstract}

Correspondence to: Professor Yingkang Shi, Department of Cardiothoracic Surgery, West China Hospital of Sichuan University, 37 Guo Xue Xiang, Chengdu, Sichuan 610000, P.R. China

E-mail: shiykwc@163.com

Professor Jun Wei, Medical Experimental Center, General Hospital of Ningxia Medical University, 840 Sheng Li Street, Yinchuan, Ningxia 750004, P.R. China

E-mail: weijny@163.com

*Contributed equally

Key words: homocysteine, fatty acid binding protein 4, DNA methylation, cholesterol accumulation cloning vector, pcDNA3.1-EGFP, to construct the recombinant plasmid, pcDNA3.1-EGFP/FABP4, which was identified using restriction endonuclease digestion analysis and DNA sequencing. The pcDNA3.1-EGFP/FABP4 expression plasmid was transfected into THP-1 monocyte-derived macrophages, mediated by liposome reagent, following which the expression levels of FABP4 were detected using RT-qPCR and western blot analyses. The present study also determined the intracellular accumulation of total cholesterol in the macrophages. The results indicated that Hcy decreased the levels of FABP4 promoter methylation, but increased the mRNA and protein expression levels of FABP4 in the macrophages, compared with the control group ( $0 \mu \mathrm{M}$ Hcy). However, no dose-dependent changes were observed with increasing concentrations of Hcy. The recombinant fluorescent eukaryotic expression vector, pcDNA3.1-EGFP/FABP4, was successfully constructed and effectively expressed in the THP-1 macrophages. The results also showed that FABP4 accelerated the accumulation of cholesterol in the macrophages. Taken together, the results of the present study suggested that FABP4 DNA hypomethylation induced by Hcy may be involved in the overexpression of FABP4, thereby inducing cholesterol accumulation in macrophages.

\section{Introduction}

Fatty acid-binding protein 4 (FABP4), also referred to as aP2 and adipocyte FABP, is expressed at high levels in adipocytes and macrophages, and is a cytosolic lipid chaperone, which regulates cellular lipid metabolism and the reception of lipid signals (1), which are actively involved in atherogenic processes. FABP4-deficiency has been shown to protect against atherosclerosis (AS) in apolipoprotein E-deficient mice, and FABP4 knockout animal models show reduced lipolysis, which offers protection from the development of AS (2). Investigations in bone marrow transplantation have also demonstrated that this atheroprotective effect of FABP4 is predominantly associated with its actions in macrophages (3). Thus, it is clear that the involvement of FABP4 is significant 
in local macrophage responses in atherogenesis, however, the precise cellular and molecular mechanisms underlying these phenomena remain to be fully elucidated. Previous studies have reported the presence of FABP4 in macrophages, and have shown that the expression of FABP4 can be induced by oxidized low density lipoprotein (oxLDL), peroxisome proliferator-activated receptor $\gamma$ (PPAR $\gamma$ ) agonists and the differentiation of monocytes to macrophages (4-6).

Homocysteine (Hcy) is a homologue of the amino acid, cysteine, differing by an additional methylene bridge (- $\mathrm{CH} 2-)$. Accumulating evidence has suggested that hyperhomocysteinemia (HHcy) is an independent risk factor of AS (7). However, the mechanisms responsible, which may be multifactorial, remain to be fully elucidated (8). Increasing evidence has indicated that Hcy may be involved in the disturbance of the expression of AS-associated genes through the interference of DNA methylation (9). The metabolism of Hcy is specifically involved in the methionine cycle for transmethylation reactions, therefore, its elevation has been implicated in the interference of DNA methylation modification and the epigenetic regulation of genes (10).

DNA methylation, the addition of a methyl group to form 5 -methylcytosine in the context of a $\mathrm{CpG}$ dinucleotide via methyltransferase, refers to a form of epigenetic modification. It has been demonstrated that aberrant DNA methylation is involved in Hcy-associated pathology, which exhibits global hypomethylation, however, hypermethylation occurs in certain genes. DNA hypermethylation leads to gene silencing, whereas hypomethylation results in gene activation in the regulatory region, together with altered binding profiles of transcription factors (11). Chronic human diseases, including AS, are either caused or affected by abnormal DNA methylation (12). Previous compelling evidence has indicated that Hcy epigenetically regulates certain specific targets, including 15-lipoxygenase, extracellular superoxide dismutase, estrogen receptor $\alpha$, cyclin A and PPARs by inhibiting DNA methylation, which in turn favors early AS $(13,14)$. Therefore, the interference of DNA methylation may be an important mechanism of Hcy-induced AS. However, whether Hcy can affect the DNA methylation and expression of FABP4 in macrophages, and further regulate cellular lipid metabolism remains to be elucidated.

Therefore, the present study hypothesized that Hcy, at a relevant concentration, affects the level of FABP4 DNA methylation and accelerates the development of AS through the accumulation of cholesterol in macrophages. Investigation to confirm this hypothesis may elucidate a potential mechanism against AS, in which epigenetic gene silencing is a feature.

\section{Materials and methods}

THP-1 cell culture and cell treatment. Human monocytic leukemia THP-1 cells (West China School of Preclinical and Forensic Medicine, Sichuan University, Sichuan, China) were cultured in RPMI 1640 medium supplemented with $15 \%(\mathrm{v} / \mathrm{v})$ heat-inactivated fetal bovine serum (GE Healthcare Life Sciences, Logan, UT, USA) in a humidified atmosphere of $5 \% \mathrm{CO}_{2}$ at $37^{\circ} \mathrm{C}$. The monocytes were initially differentiated into macrophages by the addition of $500 \mathrm{nM}$ phorbol 12-myristate 13-acetate (PMA; Sigma-Aldrich, St. Louis, MO,
USA) for $48 \mathrm{~h}$, following which the macrophages were incubated $\left(37^{\circ} \mathrm{C}, 5 \% \mathrm{CO}_{2}\right)$ in medium with different concentrations of Hcy and its antagonist for $24 \mathrm{~h}$. In the present study, the cells were divided into five groups: Cells $\left(2 \times 10^{6}\right)$ were exposed to different concentrations of Hcy $(50,100,200$ and $500 \mu \mathrm{M})$, as the Hcy group, others were incubated with Hcy $(100 \mu \mathrm{M})$ and folic acid (FA; $30 \mu \mathrm{M})$ and vitamin $\mathrm{B}_{12}\left(\mathrm{VB}_{12} ; 30 \mu \mathrm{M}\right)$, as the treatment group, and the remainder were treated without Hcy, as a control group. The cells in all groups were cultured for another $24 \mathrm{~h}$, and all cells were used for subsequent analysis.

Construction of the pcDNA3.1-EGFP/FABP4 recombinant plasmid and its transfection into THP-1 monocyte-derived macrophages. The human FABP4 gene was amplified from the cDNA of THP-1 macrophages. The human FABP4 gene and the pcDNA3.1-EGFP vector were double-digested using EcoRI and HandIII restriction endonucleases (Takara Bio Inc., Otsu, Japan), and the FABP4 gene fragment was then inserted into the pcDNA3.1-EGFP cloning vector to construct the recombinant plasmid, pcDNA3.1-EGFP/FABP4. The recombinant pcDNA3.1-EGFP/FABP4 plasmid was identified using restriction endonuclease digestion analysis and DNA sequencing. According to the manufacturer's protocol, the THP-1 macrophages grown in 6-well plates were transfected with the recombinant plasmid (pcDNA3.1-EGFP/FABP4) and empty plasmid (pcDNA3.1-EGFP), mediated by liposome reagent (Lipofectamine 2000; Invitrogen; Thermo Fisher Scientific,Inc., Waltham,MA,USA) as the recombinant plasmid group and the empty plasmid control group, respectively. The untransfeced group was set as the normal control group. The levels of green fluorescence in the cells were observed using fluorescence microscopy, and the expression levels of FABP4 were detected using reverse transcription-quantitative polymerase chain reaction (RT-qPCR) and western blot analyses.

$R T$ - $q P C R$ for the $m R N A$ expression of FABP4. Total RNA was extracted from the cultured macrophages using TRIzol reagent (Invitrogen; Thermo Fisher Scientific, Inc.), following which the RNA was reverse transcribed into cDNA using a RevertAid first strand cDNA synthesis kit (MBI Fermentas, Inc., Vilnius, Lithuania), according to the manufacturer's protocol. The cDNAs $(2 \mu \mathrm{l})$ were amplified using a SYBR Green PCR kit (MBI Fermentas, Inc.). Primer Premier 5 software (Premier Biosoft, Palo Alto, CA, USA) was used to design the primers. The primer nucleotide sequences of FABP4 (NM_001442.2; https://www.ncbi.nlm.nih.gov/nuccore/NM_001442.2) were as follows: Forward 5'-TACTGAGATTTCCTTCATACT GGGC-3' and reverse 5'-GCTCTCTCATAAACTCTCGTG GAAG-3', with a length of $372 \mathrm{bp}$. The gene expression of FABP4 was normalized to that of glyceraldehyde-3-phosphate dehydrogenase (GAPDH), forward 5'-AGAAGGCTGGGG CTCATTTG-3' and reverse 5'-AGGGGCCATCCACAG TCTTC-3'. The RT-qPCR reaction was performed using an FTC-3000 real-time PCR detection system (Funglyn, Toronto, Canada), according to the manufacturer's protocol, as follows: $94^{\circ} \mathrm{C}$ for $10 \mathrm{~min}, 50$ cycles at $94^{\circ} \mathrm{C}$ for $15 \mathrm{sec}$, at the annealing temperature of $53^{\circ} \mathrm{C}$ for $30 \mathrm{sec}$ and $72^{\circ} \mathrm{C}$ for $30 \mathrm{sec}$. The RNA level of the gene was acquired from the value of the quantification cycle $(\mathrm{Cq})$ of the RT-qPCR associated with that of GAPDH using the following formula: $\Delta \mathrm{Cq}, \Delta \mathrm{Cq}=\mathrm{Cq}_{\mathrm{GAPDH}}-\mathrm{Cq}_{\mathrm{gene}}$ 
Table I. Inner and outer primers used for $F A B P 4$ DNA methylation analysis.

\begin{tabular}{llcc}
\hline FABP4 & \multicolumn{1}{c}{ Primer sequence $\left(5^{\prime} \rightarrow 3^{\prime}\right)$} & Temperature $\left({ }^{\circ} \mathrm{C}\right)$ & Product size $(\mathrm{bp})$ \\
\hline Outer & Forward: ATTTTATTAGGGAGAGAAGGAAAAA & 71.1 \\
& Reverse: TCACATCTCAAAATCTCAAAACTAAC & 414 \\
Methylated & Forward: AAGTTGGAAGTTTTTTTTGTTAACG & 69.3 \\
& Reverse: CCTTTACCTATATTTACTTCTTTCGAA & 6 \\
Unmethylated & Forward: AGTTGGAAGTTTTTTTTGTTAATGG & 69.2 \\
& Reverse: TTTACCTATATTTACTTCTTTCAAA & 134 \\
\hline
\end{tabular}

FABP4, fatty acid binding protein 4 .

(15). The final results were expressed as $\mathrm{N}$-fold differences in the target gene expression, relative to the calibrator, termed ' $\mathrm{N}_{\text {target }}$ ', which was determined as follows: $\mathrm{N}_{\text {target }}=2^{\Delta \text { Cqsample }-}$ $\Delta$ Cqcalibrator, where the $\Delta \mathrm{Cq}$ values of the calibrator and sample were determined by subtracting the $\mathrm{Cq}$ value of the target gene from the $\mathrm{Cq}$ value of GAPDH, as previously described (11).

Western blot analysis for FABP4. The cultured macrophages were harvested by scraping with a plastic scraper and washing with phosphate-buffered saline (PBS). The protein was extracted using a protein extraction kit (Nanjing KeyGen Biotech Co., Ltd., Nanjing, China), and protein concentration was determined using a Bicinchoninic Acid Protein Assay kit (Nanjing KeyGen Biotech Co., Ltd.). Equal quantities of protein $(80 \mu \mathrm{g})$ and a known molecular weight marker were loaded onto $12 \%$ sodium dodecyl sulfate-polyacrylamide gels (Nanjing KeyGen Biotech Co., Ltd.) and were transferred onto a polyvinylidene fluoride membrane (EMD Millipore, Boston, MA, USA) by electrophoresis at $300 \mathrm{~mA}$ for $50 \mathrm{~min}$ at $4^{\circ} \mathrm{C}$. The membrane was then blocked in $10 \mathrm{ml} 5 \%$ skimmed milk for $2 \mathrm{~h}$ at room temperature with gentle agitation on a platform shaker. The blocked membranes was respectively incubated with a rabbit monoclonal anti-FABP4 antibody (cat. no. MABS172; 1:400 dilution; EMD Millipore) and $\beta$-actin antibody (cat. no. TA-09; 1:2,000 dilution; ZSGB-BIQ, Beijing, China) in $10 \mathrm{ml}$ primary antibody dilution buffer overnight at $4^{\circ} \mathrm{C}$. The membrane was then washed three times for $10 \mathrm{~min} /$ wash with PBS-0.1\% Tween-20 (PBST) and incubated with secondary antibody (cat. no. ab6721; goat anti-rabbit horseradish peroxidase-IgG; Abcam, Cambridge, MA, USA) in PBS at 1:2,000 dilution for $2 \mathrm{~h}$ at room temperature. Following being washed three times with PBST, the FABP4 on the membrane was incubated with enhanced chemiluminescence (ECL; Elabscience Biotechnology Co., Ltd., Wuhan, China) for $1 \mathrm{~min}$ at room temperature, following which the excess ECL solution was drained, and the membrane was wrapped in a plastic wrap and exposed to X-ray film (Kodak, Shanghai, China). The densities of the bands were quantified and the relative values were normalized to that of $\beta$-actin using the following formula: Associated value $=$ experimental densitometry value $/ \beta$-actin value).

Nested touchdown methylation specific-PCR (ntMSP) analysis of FABP4 DNA methylation. Genomic DNA from the macrophages was isolated using a Wizard Genomic DNA Purification kit (Promega, Madison, WI, USA) and modified using an EZ DNA Methylation-Gold ${ }^{\mathrm{TM}}$ kit (Zymo Research Corp., Orange, CA, USA), following which it was used as a template for ntPCR. Following bisulfite modification of the genomic DNA, unmethylated cytosine residues were converted into uracil. The FABP4 DNA methylation primers for MSP amplification were designed using the MethPrimer bioinformatics program (http://www.urogene.org/methprimer/index.html). The inner and outer primers were designed and synthesized based on the DNA sequences of FABP4 (Table I).

To reduce mispriming and to increase efficiency, ntMSP was used for amplification, which comprised a two-step PCR amplification procedure following the standard sodium bisulfite DNA modification. In accordance with the specification requirements of DNA remodified sodium, $4 \mu \mathrm{l}$ of modified DNA was obtained, $1 \mu \mathrm{l}$ upstream and $1 \mu \mathrm{l}$ downstream of each primer, using Go Taq Colorless Master mix (x2; $12.5 \mu \mathrm{l}$; Promega), with nuclease-free water added to $25 \mu \mathrm{l}$. The samples were subjected to the following steps in a thermal cycler: $94^{\circ} \mathrm{C}$ for $5 \mathrm{~min}, 94^{\circ} \mathrm{C}$ for $30 \mathrm{sec}, 74^{\circ} \mathrm{C}$ for $30 \mathrm{sec}$ and $72^{\circ} \mathrm{C}$ for $1 \mathrm{~min}$, for 30 cycles, decreasing $0.5^{\circ} \mathrm{C}$ every cycle, $94^{\circ} \mathrm{C}$ for $30 \mathrm{sec}$, for $59^{\circ} \mathrm{C} 30 \mathrm{sec}$ and $72^{\circ} \mathrm{C}$ for $1 \mathrm{~min}, 20$ cycles, and $72^{\circ} \mathrm{C}$ for $7 \mathrm{~min}$. The PCR products were separated by electrophoresis on $2 \%$ agarose gel. The bands were visualized under ultraviolet illumination and calculated using the following formula: Methylation $(\%)=$ methylation / (methylation + unmethylation) $\times 100 \%$. Each sample was examined using ntMSP analysis three times.

Assessment of intracellular total cholesterol (TC). The macrophages were scraped off the culture plate using a rubber scraper and washed in PBS three times, following which they were re-suspended in $0.5 \mathrm{ml}$ PBS ( $\mathrm{pH} 7.4$ ) and lysed by ultrasound for $1 \mathrm{~min}$. The TC was determined, according to the manufacturer's protocol of the Total cholesterol kit-CHO (Beijing BHKT Clinical Reagent Co., Ltd, Beijing, China). TC concentrations were expressed as $\mathrm{mM} / 1$.

Statistical analysis. Experiments were performed at least three times and the results are expressed as the mean \pm standard error of the mean. The data were analyzed using one-way analysis of variance, and additional analysis was performed using the Student-Newman-Keuls test for multiple comparisons within different groups. GraphPad Prism 5.01 software was used for analysis (version 5.01, GraphPad Software, Inc., 
A

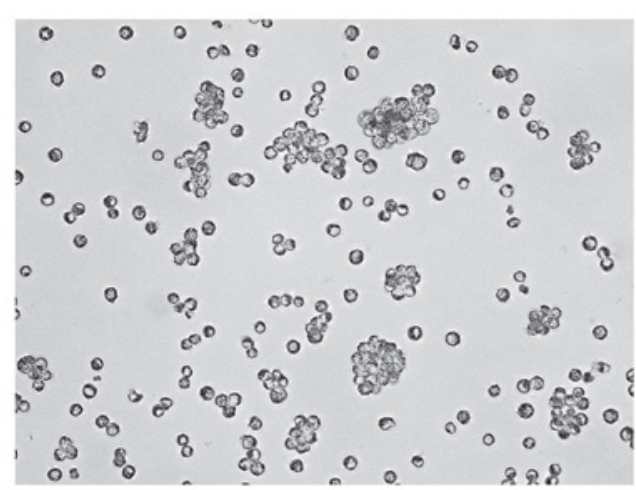

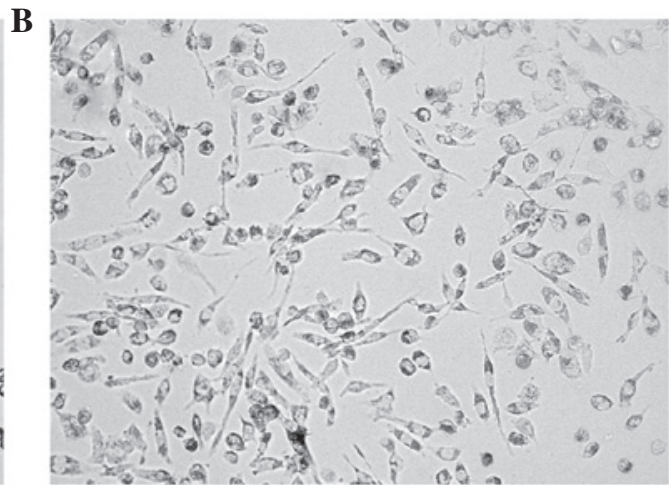

Figure 1. Formation of macrophages derived from THP-1 monocytes induced by PMA. (A) THP-1 monocytes were incubated for 48 h with PMA (500 nM). (B) THP-1 monocytes were incubated without PMA $(500 \mathrm{nM})$. Images of the cells were captured at $\mathrm{x} 200$ magnification under a phase-contrast inverted microscope. PMA, phorbol 12-myristate 13-acetate.

La Jolla, CA, USA). P<0.05 was considered to indicate a statistically significant difference.

\section{Results}

Formation of macrophages derived from activated THP-1 monocytes. The THP-1 monocytes were observed to grow in suspension and not adhere to the plastic surfaces of the culture flasks (Fig. 1A). For the induction of terminal differentiation into macrophages, the THP-1 cells were cultured in the presence of 500 nM PMA for 2 days, and then co-cultured with 50, 100,200 and $500 \mathrm{mM} \mathrm{Hcy}$ or $100 \mathrm{mM} \mathrm{Hcy}+\mathrm{VB}_{12}+\mathrm{FA}$ for $24 \mathrm{~h}$. Following $72 \mathrm{~h}$ of culture, the cells had adhered and spread across the bottom of the dish, and had the morphological characteristics of macrophages (Fig. 1B).

Hcy-induced upregulation of the $M R N A$ and protein expression levels of FABP4. To investigate whether the regulation of FABP4 occurred in the context of Hcy, the present study examined the mRNA and protein expression levels of FABP4 using RT-qPCR and western blot analyses in macrophages exposed to Hcy for $24 \mathrm{~h}$. The data demonstrated that the expression of FABP4 was substantially upregulated following incubation with Hcy for $24 \mathrm{~h}$ at various concentrations (between 50 and $500 \mu \mathrm{M}$ ), and the mean mRNA expression levels of FABP4 in the experimental groups $(50,100,200$ and $500 \mathrm{mM} \mathrm{Hcy}$, and $100+\mathrm{VB}_{12}+\mathrm{FA}$ ) were $10.84,11.46,5.89,3.45$ and 1.41 times higher, compared with that in the control group, respectively (Fig. 2A). The highest level of expression was observed at the concentration of $100 \mathrm{mM} \mathrm{Hcy}(\mathrm{P}<0.01)$. In addition, the relative mRNA expression level of FABP4 in the $100+\mathrm{VB}_{12}+\mathrm{FA}$ group decreased significantly $(87.69 \%)$ compared with the $100 \mu \mathrm{M}$ Hcy group $(\mathrm{P}<0.01)$. The effects of Hcy on the protein levels of FABP4 (Fig. 2B) were measured using western blot analysis, which showed similar results to the mRNA levels. The protein expression levels of FABP4 in the 100 and $200 \mu \mathrm{M}$ Hcy groups were 3.27 and 2.64 times higher, compared with that in the control group $(\mathrm{P}<0.05)$, respectively. $\mathrm{FA}$ and $\mathrm{VB}_{12}$ also affected the protein expression of FABP4 significantly, and the relative protein expression of FABP4 in the $100+\mathrm{VB}_{12}+\mathrm{FA}$ group was significantly decreased by $52.52 \%$, compared with that in the $100 \mu \mathrm{M}$ Hcy group $(\mathrm{P}<0.05)$. However, as the concentrations of Hcy increased, no dose-dependent increase in the mRNA or protein levels of FABP4 were observed. These results indicated that Hcy affected the expression of FABP4 at the transcriptional and translational levels.

Hcy-induced FABP4 DNA hypomethylation in THP-1 macrophages. DNA methylation patterns have been considered a useful molecular marker for AS, and Hcy can have a global effect on DNA methylation and activate various target genes (16). To determine the effects of Hcy on FABP4 DNA methylation and investigate DNA methylation in the promoter region of FABP4, the THP-1 monocyte-derived macrophages in the present study were treated with Hcy at various concentrations for $24 \mathrm{~h}$, and the DNA methylation status of the FABP4 promoter region of the THP-1 macrophages was determined via ntMSP. As shown in Fig. 3, an increase in the concentration of Hcy between 50 and $100 \mathrm{mM}$, and a further increase to $500 \mathrm{mM}$, led to statistically significant differences in the levels of FABP4 promoter region DNA methylation, compared with the control group. The methylation changes in the $100 \mathrm{mM}$ group were the most marked. It was also found that, in the experimental groups $(50,100,200$ and $500 \mathrm{mM}$ $\mathrm{Hcy}$, and $\left.100+\mathrm{VB}_{12}+\mathrm{FA}\right)$, the levels of FABP4 DNA methylation decreased significantly, by 18.84, 43.38, 23.65, 16.50 and $25.28 \%$, respectively. The level of FABP4 DNA methylation in the $100+\mathrm{VB}_{12}+\mathrm{FA}$ group increased significantly (31.97\%), compared with the $100 \mathrm{mM}$ Hcy group $(\mathrm{P}<0.01$; Fig. 3). These results indicated that Hcy decreased the level of FABP4 promoter region DNA methylation.

Hcy-induced cellular TC accumulation in THP-1 macrophages. The present study also assessed whether Hcy accelerated cholesterol accumulation in the macrophages. The human THP-1 macrophages were treated with increasing concentrations of Hcy, and intracellular TC accumulation was assessed using a Total Cholesterol kit. Hcy significantly increased intracellular TC accumulation, compared with the control group, and the highest level was observed at a concentration of $100 \mu \mathrm{M}$ Hcy $(\mathrm{P}<0.01)$. However, the Hcy-induced increase in intracellular TC accumulation did not occur in a dose-dependent manner (Fig. 4).

Identification of the inserted constructed recombinant pcDNA3.1-EGFP/FABP4 plasmid. The recombinant vector 
A

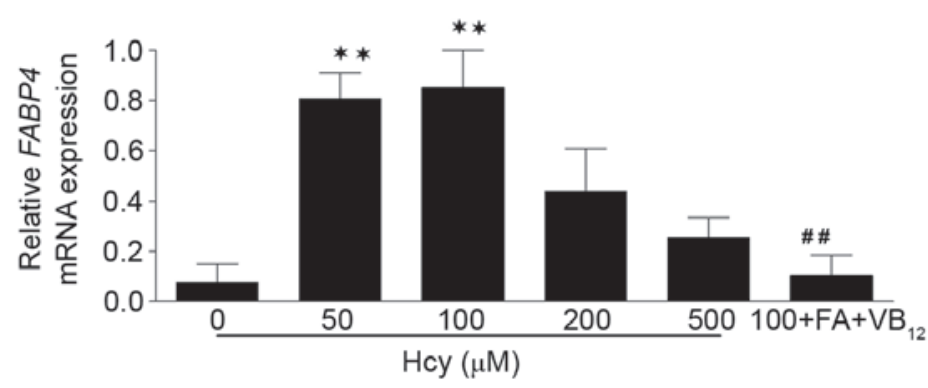

$\mathbf{B}$
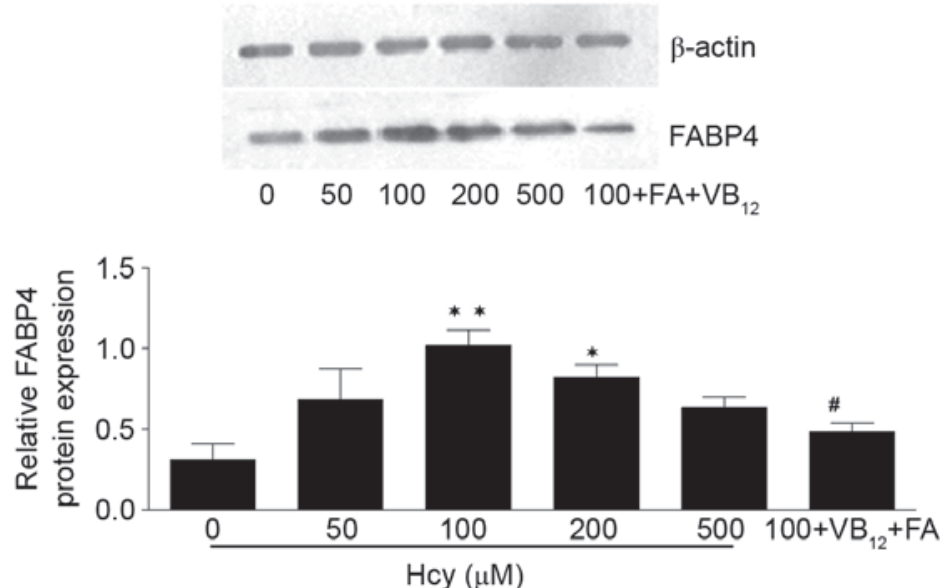

Figure 2. mRNA and protein expression levels of FABP4 in THP-1 monocyte-derived macrophages. (A) mRNA expression levels of FABP4, acquired from the $\mathrm{Cq}$ values of reverse transcription-quantitative polymerase chain reaction analysis, relative to GAPDH and expressed as N-fold differences in the expression of FABP4 relative to the calibrator, termed 'Ngene'. Data are expressed as the mean \pm standard error of the mean. (B) Effect of Hcy on the protein levels of FABP4 were measured by western blotting. The immunoblots were analyzed by densitometry. Protein samples were prepared from THP-1 monocyte-derived macrophages. Protein signals were visualized by electrochemiluminescence using Quantity One software. Relative values were normalized to the corresponding $\beta$-actin band intensities, The protein levels of FABP4 in the macrophages incubated with Hcy were significantly increased, similar to the mRNA levels of FABP4. These results suggested that the macrophages cultured with Hcy for $24 \mathrm{~h}$ produced increased FABP4. ${ }^{*} \mathrm{P}<0.05$ and ${ }^{* *} \mathrm{P}<0.01$, vs. control; ${ }^{*} \mathrm{P}<0.05$ and ${ }^{\# \#} \mathrm{P}<0.01$, vs. $100 \mu \mathrm{M}$ Hcy. FABP4, fatty acid binding protein 4; Hcy, homocysteine; 100+FA+VB12, $30 \mu \mathrm{M}$ folate $+30 \mu \mathrm{M}$ vitamin $\mathrm{B}_{12}$, dissolved in $100 \mu \mathrm{M}$ Hcy.

encoding FABP4 was constructed, and it was confirmed that the human $F A B P 4$ fragment had been successfully inserted into the pcDNA3.1-EGFP fluorescent eukaryotic expression vector. The FABP4 PCR products were 412 bp on the $1.5 \%$ agarose gel (Fig. 5A). The 412 bp fragment encoding human FABP4 was inserted into the pcDNA3.1-EGFP plasmid between the EcoRI and HindIII sites. The successfully constructed recombinant plasmid, pcDNA3.1-EGFP/FABP4, was cut into two fragments using EcoRI and HindIII (Fig. 5B). In addition, the results of the DNA sequencing were identical to the FABP4 sequence reported on GenBank confirming the results.

Transient FABP4 recombinant transfection induces overexpression of the $m R N A$ and protein levels of FABP4 in THP-1 macrophages. The recombinant expression plasmid containing the FABP4 gene was successfully transfected into the THP-1 macrophages, and the effective mRNA and protein expression of FABP4 were also confirmed using RT-qPCR and western blot analyses, respectively. The data demonstrated that the expression of FABP4 was significantly upregulated in the recombinant plasmid group, with the mean mRNA expression of FABP4 in the recombinant plasmid group being 3.90 and 3.85 times higher than those in the normal control group and empty plasmid control group, respec- tively $(\mathrm{P}<0.01$; Fig. $6 \mathrm{~A})$. The protein levels of FABP4 were measured using western blot analysis, which showed similar results to those observed for mRNA expression (Fig. 6B). The protein expression of FABP4 in the recombinant plasmid group was higher, compared with the normal control group and empty plasmid control group, (69.79 and 63.36\%, respectively; $\mathrm{P}<0.01)$. Therefore, the pcDNA3.1-EGFP/FABP4 recombinant fluorescent eukaryotic expression vector was successfully constructed and effectively expressed in the THP-1 macrophages, which provided an experimental basis for further experiments.

Effect of the FABP4-specific recombinant on intracellular TC contents. The present study subsequently investigated whether FABP4 accelerated cholesterol accumulation in the macrophages. The human THP-1 macrophages were transfected with the pcDNA3.1-EGFP plasmid and pcDNA3.1-EGFP/FABP4 recombinant plasmid, respectively, and TC levels were examined. As shown in Fig. 7, intracellular TC accumulation in the recombinant plasmid group was significantly higher, compared with that in the normal control group and empty plasmid control group $(\mathrm{P}<0.01)$. These results indicated that the overexpression of FABP4 significantly increased intracellular TC accumulation. These observations were consistent with a 
A

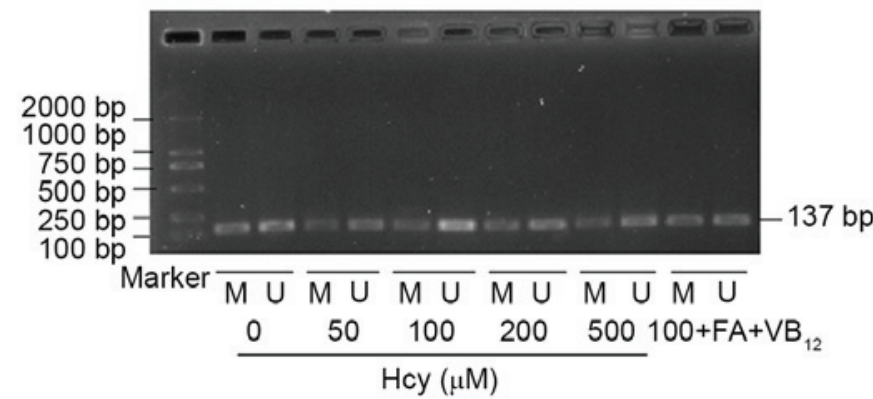

$\mathbf{B}$

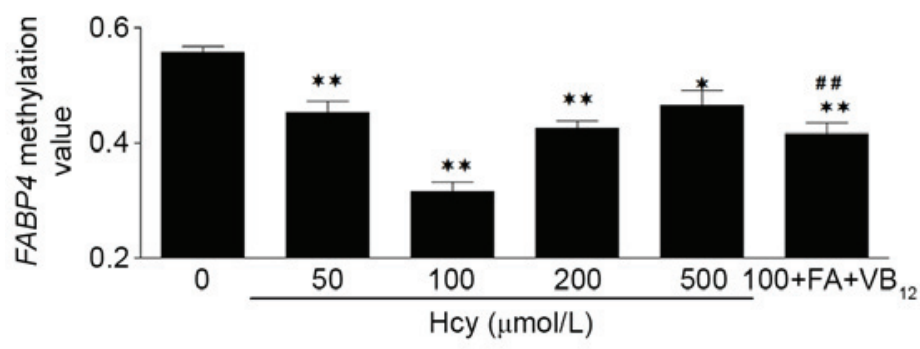

Figure 3. Effects of Hcy on FABP4 DNA methylation in THP-1 monocyte-derived macrophages. The methylation status of FABP4 was detected using nested touchdown methylation-specific PCR following bisulfite modification of the DNA from THP-1 macrophages. (A) Representative electrophoresis bands. (B) Promoter methylation levels of FABP4 were calculated using densitometry. Values are expressed as the mean \pm standard error of the mean. "P $<0.05$ and ${ }^{* *} \mathrm{P}<0.01$, vs. control group; ${ }^{\# /} \mathrm{P}<0.01$, vs. $100 \mu \mathrm{M}$ Hcy group. PCR, polymerase chain reaction; FABP4, fatty acid binding protein 4; Hcy, homocysteine; $100+\mathrm{FA}+\mathrm{VB}_{12}, 30 \mu \mathrm{M}$ folate $+30 \mu \mathrm{M}$ vitamin $\mathrm{B}_{12}$, dissolved in $100 \mu \mathrm{M} \mathrm{Hcy} ; \mathrm{M}$, methylated product; $\mathrm{U}$, uunmethylated product.

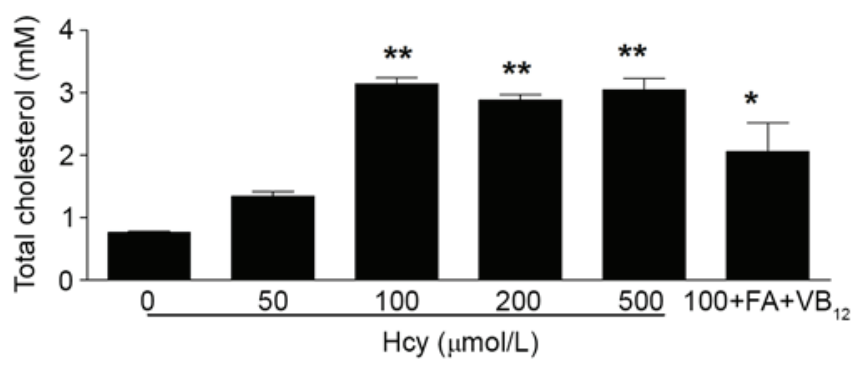

Figure 4. Levels of TC in THP-1 monocyte-derived macrophages. The levels of TC were detected using a Total Cholesterol kit. The levels of TC were significantly increased by Hcy, with the highest value at $100 \mu \mathrm{M} \mathrm{Hcy,com-}$ pared with the control group $(\mathrm{P}<0.01)$. Increasing Hcy concentrationss did not cause a dose-dependent increase in TC content. The data are expressed as the mean \pm standard error of the mean. ${ }^{*} \mathrm{P}<0.05$ and ${ }^{* *} \mathrm{P}<0.01$, vs. control group. Hcy, homocysteine; $100+\mathrm{FA}+\mathrm{VB}_{12}, 30 \mu \mathrm{M}$ folate $+30 \mu \mathrm{M}$ vitamin $\mathrm{B}_{12}$ dissolved in $100 \mu \mathrm{M} \mathrm{Hcy}$. TC, total cholesterol.

previous report which suggested a critical role for FABP4 in macrophage lipid accumulation (17).

\section{Discussion}

Previous reports have demonstrated that elevated levels of Hcy have been associated with a number of disease states, including AS $(18,19)$. Despite the important contribution of Hcy to accelerated AS, the specific molecular mechanisms in response to Hcy within macrophages, which is important in atherogenesis, remain to be fully elucidated.

FABP4 is a $15 \mathrm{kD}$ cytoplasmic protein binding hydrophobic ligands. Deficiency of FABP4 enhances CD36-mediated lipoprotein entry and, at the same time, activates ABCA1-dependent lipid efflux to a greater extent, thereby lowering intracellular lipid contents (20). In the present study, it was found that the exposure of THP-1 macrophages to Hcy was associated with a significant elevation in the expression of FABP4, reaching its maximum level when $100 \mu \mathrm{M}$ Hcy was used. It was also found that the enhanced expression of FABP4 was paralleled with increases in intracellular TC levels. The present study demonstrated that the exposure of macrophages to Hcy appeared to increase intracellular FAPB4 levels and lipid contents, similar to observations in the transformation of macrophages into foam cells in the presence of ox-LDL stimulation (21). In addition, Hcy can be recycled into methionine or converted into cysteine with the assistance of FA and $\mathrm{VB}_{12}(22)$, and mild doses of Hcy inhibitors, including $\mathrm{VB}_{12}$ and FA, markedly attenuated FABP4-induced macrophage lipid accumulation. These findings indicated that the Hcy inhibitors suppressed exclusively pathological excessive lipid accumulation in the macrophages.

To further understand the role of FABP4, the present study constructed the pcDNA3.1-EGFP/FABP4 recombinant vector and successfully transduced the FABP4 gene into macrophages derived from THP-1 monocytes. The increased expression of FABP4 from the transfected macrophages was confirmed using RT-qPCR and western blot analyses. In addition, cellular TC accumulation was examined, and it was found that the intracellular TC content in the FABP4 recombinant plasmid group was also significantly increased. Thus, the increase in cellular TC accumulation was accelerated by the enhanced expression of FABP4, either directly or indirectly. These results suggested that FABP4 may be a potential therapeutic target for the treatment of AS.

Previous population studies have revealed that genetic variations at the FABP4 locus in humans lead to lowered serum triglyceride levels, and a markedly reduced risk of 

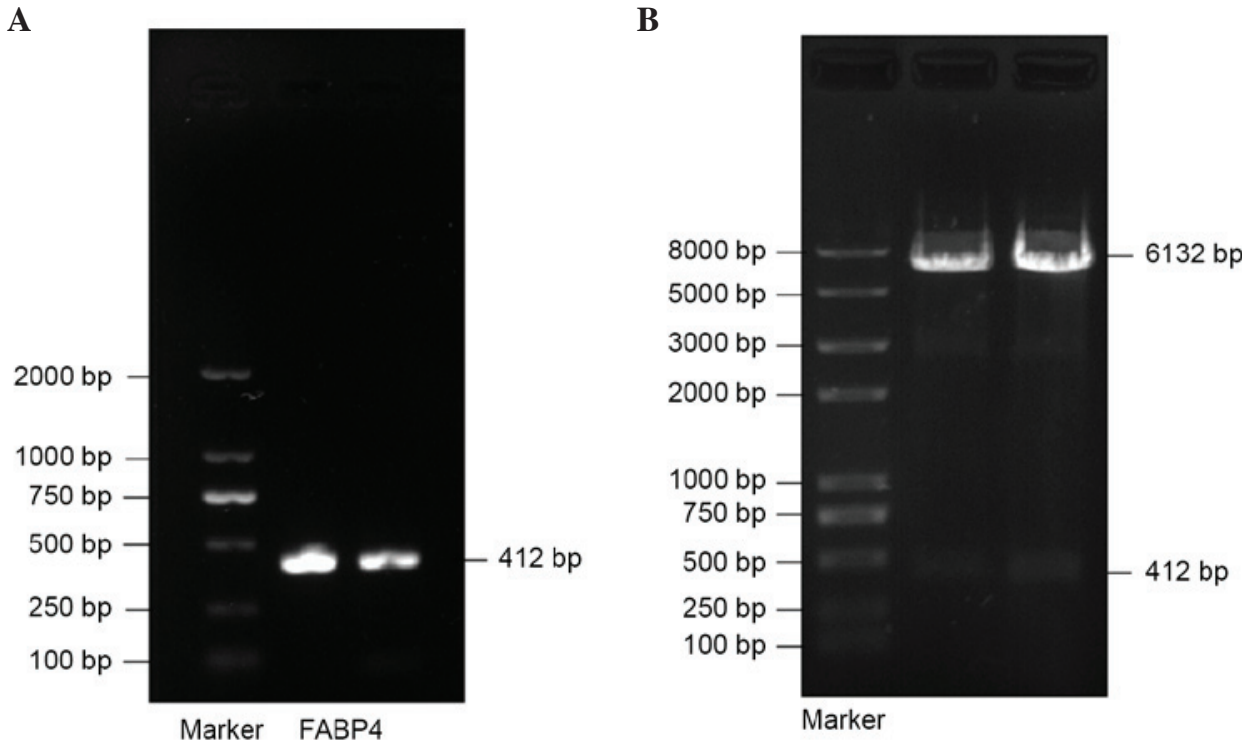

Figure 5. Identification of the constructed recombinant plasmid, pcDNA3.1-EGFP/FABP4, by PCR amplification and restriction endonuclease digestion analyses. Successful construction of the recombinant plasmid, pcDNA3.1-EGFP/FABP4, was confirmed by (A) PCR amplification and (B) double digestion with EcoRI and HindIII of the recombinant plasmid. FABP4, fatty acid binding protein 4; PCR, polymerase chain reaction.

A

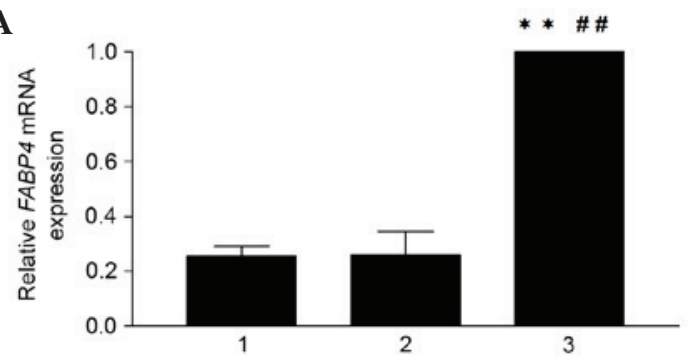

B
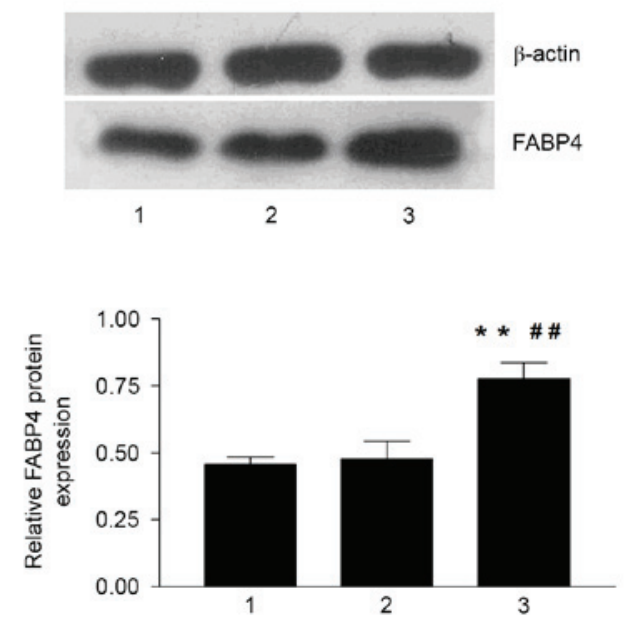

Figure 6. Effect of transient transfection of THP-1 macrophages on the expression of FABP4. Macrophages were transfected with the empty plasmid (pcDNA3.1-EGFP) or recombinant plasmid (pcDNA3.1-EGFP/FABP4), respectively. (A) mRNA expression levels of FABP4 were determined using reverse transcription-quantitative polymerase chain reation analysis. Data are expressed relative to untreated macrophages (normal control group), arbitrarily set at the level of 1 , and are presented as the mean \pm standard error of the mean of at least three independent experiments. (B) Protein expression levels of FABP4 in each group were analyzed using western blot analysis. Relative values were normalized to the corresponding $\beta$-actin band intensities. The results shown represent the average densitometric analysis of three independent experiments. Data are expressed in arbitrary units. 1, normal control group; 2, empty plasmid control group; 3, recombinant plasmid group. ${ }^{* *} \mathrm{P}<0.01$, vs. normal control group; ${ }^{\# \#} \mathrm{P}<0.01$, vs. empty plasmid group. FABP4, fatty acid binding protein 4 .

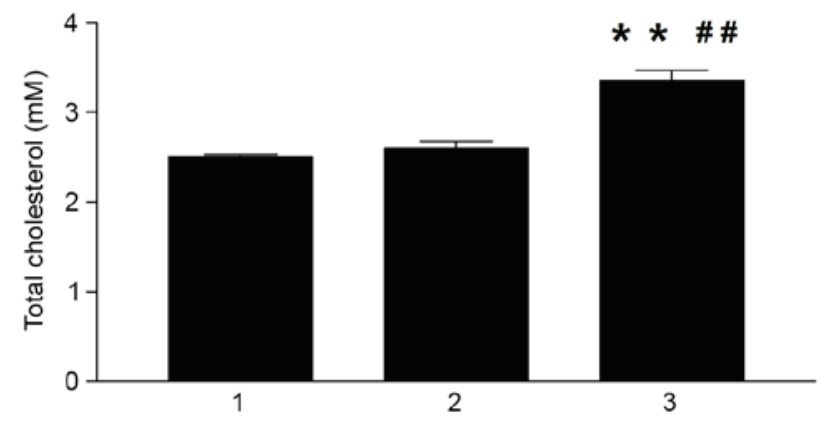

Figure 7. Assessment of intracellular TC content in THP-1 macrophages. Quantification of the intracellular TC levels was performed according to the manufacture's protocols of the Total Cholesterol kit and analyzed on a microplate reader. 1, normal control group; 2, empty plasmid control group; 3 , recombinant plasmid group. Data are presented as the mean \pm standard error of the mean. ${ }^{* *} \mathrm{P}<0.01$, vs. normal control group; ${ }^{\# \#} \mathrm{P}<0.01$, vs. empty plasmid group. TC, total cholesterol.

coronary heart disease $(23,24)$. However, the exact molecular mechanisms remain to be elucidated. In our previous study, Hcy was found to be involved in the disturbance of the expression of the LDL-receptor promoter region and global genome DNA hypomethylation through the interference of DNA methylation (25-27). Increasing evidence also suggests that aberrant DNA methylation around promoter regions can lead to the development of AS (28). Therefore, the present study investigated the epigenetic mechanisms by which the elevated levels of FAPB 4 and lipids were affected by Hcy. The resulting data showed tha thet FABP4 promoter was hypomethylated following exposure to Hcy. These results indicated a causal association between the expression of FABP4 and the changes in DNA methylation, and the epigenetic mechanism of Hcy-induced AS was ascertained.

In conclusion, the results of the present study demonstrated that Hcy not only promoted the secretion of FABP4, but also induced FABP4 gene DNA hypomethylation in cultured 
human THP-1 monocyte-derived macrophages. The invasion of monocytes into the arterial wall and their subsequent differentiation into cholesterol-laden macrophages is a central feature of AS (29). Therefore, the present study examined the effects of Hcy on FABP4, and demonstrated a causal molecular link between Hcy and FABP4 in macrophage lipid accumulation. The most notable finding of the present study was that, Hcy was a stimulator of FABP4 DNA methylation in cultured macrophages, a critical cell in atherogenesis, which induced FABP4 DNA hypomethylation. The results of the present study also confirmed the mechanism that the overexpression of FABP4 accelerates TC accumulation, and thereby promotes the atherosclerotic process, Further investigations, including the analysis of lipid trafficking in macrophages from FABP4-deficient mice and inhibition of lipoprotein transporters by specific small interfering RNA or antibodies, are required to further elucidate the mechanisms.

\section{Acknowledgements}

This study was supported by grants from the National Natural Science Foundation of China (grant nos. 80160105, 81360052, 81460121, 81360027, 81360073 and 81260063) and the Advantages Besides Construction Projects of Ningxia Medical University 2014 (grant nos. XY201415 and Ningxia Medical University [2014] 062).

\section{References}

1. Furuhashi M and Hotamisligil GS: Fatty acid-binding proteins: Role in metabolic diseases and potential as drug targets. Nat Rev Drug Disc 7: 489-503, 2008.

2. Cabré A, Babio N, Lázaro I, Bulló M, Garcia-Arellano A, Masana L and Salas-Salvadó J: FABP4 predicts atherogenic dyslipidemia development. The PREDIMED study. Atherosclerosis 222: 229-234, 2012.

3. Furuhashi M, Ishimura S, Ota H and Miura T: Lipid chaperones and metabolic inflammation. Int J Inflam 2011: 642612, 2011

4. Fu Y, Luo N and Lopes-Virella MF: Oxidized LDL induces the expression of ALBP/aP2 mRNA and protein in human THP-1 macrophages. J Lipid Res 41: 2017-2023, 2000.

5. Pelton PD, Zhou L, Demarest KT and Burris TP: PPARgamma activation induces the expression of the adipocyte fatty acid binding protein gene in human monocytes. Biochem Biophys Res Commun 261: 456-458, 1999.

6. Fu Y, Luo N, Lopes-Virella MF and Garvey WT: The adipocyte lipid binding protein (ALBP/aP2) gene facilitates foam cell formation in human THP-1 macrophages. Atherosclerosis 165: 259-269, 2002.

7. Tehlivets O: Homocysteine as a risk factor for atherosclerosis: Is its conversion to s-adenosyl-L-homocysteine the key to deregulated lipid metabolism? J Lipids 2011: 702853, 2011.

8. Li L, Xie J, Zhang M and Wang S: Homocysteine harasses the imprinting expression of IGF2 and $\mathrm{H} 19$ by demethylation of differentially methylated region between IGF2/H19 genes. Acta Biochim Biophys Sin (Shanghai) 41: 464-471, 2009.

9. Yi-Deng J, Tao S, Hui-Ping Z, Jian-Tuan X, Jun C, Gui-Zhong L and Shu-Ren W: Folate and ApoE DNA methylation induced by homocysteine in human monocytes. DNA Cell Biol 26: 737-744, 2007.

10. Ma S, Zhang H, Sun W, Gong H, Wang Y, Ma C, Wang J, Cao C, Yang X, Tian J, Jiang Y: Hyperhomocysteinemia induces cardiac injury by up-regulation of p53-dependent Noxa and Bax expression through the p53 DNA methylation in ApoE(-/-) mice. Acta Biochim Biophys Sin (Shanghai) 45: 391-400, 2013.

11. Deng G, Nguyen A, Tanaka H, Matsuzaki K, Bell I, Mehta KR, Terdiman JP, Waldman FM, Kakar S, Gum J, et al: Regional hypermethylation and global hypomethylation are associated with altered chromatin conformation and histone acetylation in colorectal cancer. Int J Cancer 118: 2999-3005, 2006.
12. Subramanyam MA, Diez-Roux AV, Pilsner JR, Villamor E, Donohue KM, Liu Y and Jenny NS: Social factors and leukocyte DNA methylation of repetitive sequences: The multi-ethnic study of atherosclerosis. PLoS One 8: e54018, 2013.

13. Sharma P, Senthil kumar RD, Brahmachari V, Sundaramoorthy E, Mahajan A, Sharma A and Sengupta S: Mining literature for a comprehensive pathway analysis: A case study for retrieval of homocysteine related genes for genetic and epigenetic studies. Lipids Health Dis 5: 1, 2006.

14. Yideng J, Zhihong L, Jiantuan X, Jun C, Guizhong L and Shuren W: Homocysteine-mediated PPARalpha, gamma DNA methylation and its potential pathogenic mechanism in monocytes. DNA Cell Biol 27: 143-150, 2008.

15. Jiang Y, Zhang H, Sun T, Wang J, Sun W, Gong H, Yang B, Shi Y and Wei J: The comprehensive effects of hyperlipidemia and hyperhomocysteinemia on pathogenesis of atherosclerosis and DNA hypomethylation in ApoE-/- mice. Acta Biochim Biophys Sin 44: 866-875, 2012

16. Liang Y, Yang X, Ma L, Cai X, Wang L, Yang C, Li G, Zhang M, Sun W and Jiang Y: Homocysteine-mediated cholesterol efflux via ABCA1 and ACAT1 DNA methylation in THP-1 monocyte-derived foam cells. Acta Biochim Biophys Sin (Shanghai) 45: 220-228, 2013.

17. Wang XQ, Yang K, He YS, Lu L and Shen WF: Receptor mediated elevation in FABP4 levels by advanced glycation end products induces cholesterol and triacylglycerol accumulation in THP-1 macrophages. Lipids 46: 479-486, 2011.

18. Veeranna V, Zalawadiya SK, Niraj A, Pradhan J, Ference B, Burack RC, Jacob S and Afonso L: Homocysteine and reclassification of cardiovascular disease risk. J Am Coll Cardiol 58: 1025-1033, 2011

19. Yang AN, Zhang HP, Sun Y, Yang XL, Wang N, Zhu G, Zhang $\mathrm{H}, \mathrm{Xu} \mathrm{H}$, Ma SC, Zhang Y, Li GZ, Jia YX, Cao J and Jiang YD: High-methionine diets accelerate atherosclerosis by HHcy-mediated FABP4 gene demethylation pathway via DNMT1 in ApoE(-/) mice. FEBS Lett 589: 3998-4009, 2015.

20. Makowski L, Brittingham KC, Reynolds JM, Suttles J and Hotamisligil GS: The fatty acid-binding protein, aP2, coordinates macrophage cholesterol trafficking and inflammatory activity. Macrophage expression of aP2 impacts peroxisome proliferator-activated receptor gamma and IkappaB kinase activities. J Biol Chem 280: 12888-12895, 2005.

21. Fu Y, Luo N and Lopes-Virella MF: Oxidized LDL induces the expression of ALBP/aP2 mRNA and protein in human THP-1 macrophages. J Lipid Res 41: 2017-2023, 2000.

22. Miller AL: The methionine-homocysteine cycle and its effects on cognitive diseases. Altern Med Rev 8: 7-19, 2003.

23. Tuncman G, Erbay E, Hom X, De Vivo I, Campos H, Rimm EB and Hotamisligil GS: A genetic variant at the fatty acid-binding protein aP2 locus reduces the risk for hypertriglyceridemia, type 2 diabetes, and cardiovascular disease. Proc Natl Acad Sci USA 103: 6970-6975, 2006.

24. Furuhashi M, Fuseya T, Murata M, Hoshina K, Ishimura S, Mita T, Watanabe Y, Omori A, Matsumoto M, Sugaya $\mathrm{T}$, et al: Local production of fatty acid-binding protein 4 in epicardial/perivascular fat and macrophages is linked to coronary atherosclerosis. Arterioscler Thromb Vasc Biol 36: 825-834, 2016.

25. Huang Y, Peng K, Su J, Huang Y, Xu Y and Wang S: Different effects of homocysteine and oxidized low density lipoprotein on methylation status in the promoter region of the estrogen receptor a gene. Acta Biochim Biophys Sin (Shanghai) 39: 19-26, 2007.

26. Yideng J, Jianzhong Z, Ying H, Juan S, Jinge Z, Shenglan W, Xiaoqun $\mathrm{H}$ and Shuren W: Homocysteine-mediated expression of SAHH, DNMTs, MBD2, and DNA hypomethylation potential pathogenic mechanism in VSMCs. DNA Cell Biol 26: 603-611, 2007.

27. Jiang $\mathrm{Y}$, Sun $\mathrm{T}$, Xiong J, Cao J, Li G and Wang S: Hyperhomocysteinemia-mediated DNA hypomethylation and its potential epigenetic role in rats. Acta Biochim Biophys Sin (Shanghai) 39: 657-667, 2007.

28. Zaina S, Lindholm MW and Lund G: Nutrition and aberrant DNA methylation patterns in atherosclerosis: More than just hyperhomocysteinemia? J Nutr 135: 5-8, 2005.

29. Moore KJ and Freeman MW: Targeting innate immunity for CV benefit. Drug Discov Today Ther Strateg 5: 15-23, 2008. 